Brustkrebs

\section{Stresshormone fördern die Metastasierung}

Obradović MM et al. Glucocorticoids promote breast cancer metastasis. Nature 2019; 567: 540-544. doi:10.1038/s41586-019-1019-4

Schon lange wird angenommen, dass Stress zum Krebswachstum beiträgt. Forschende aus Basel haben nun die molekularen Mechanismen aufgedeckt, die Brustkrebsmetastasen mit erhöhten Stresshormonen verbinden. Weiter haben sie herausgefunden, dass synthetische Derivate von Stresshormonen, die häufig als entzündungshemmende Mittel in der Krebstherapie eingesetzt werden, die Wirksamkeit der Chemotherapie verringern können.

Ein großes Hindernis bei der Behandlung von metastasierendem Brustkrebs ist das Phänomen der Tumorheterogenität. Das heißt, im Verlauf der Erkrankung wird der Tumor vielfältiger und die wachsenden Unterschiede zwischen den einzelnen Krebszellen können zu einem unzureichenden Therapieerfolg führen.

Da die zugrunde liegenden Mechanismen dieses Phänomens unklar waren, untersuchte die Forschungsgruppe von der Universität und dem Universitätsspital Basel die Zellen einer bestimmten Krebsform, die häufig metastasiert, des sog. 3-fach negativen Brustkrebses. Diese Krebsart ist resistent gegen Standardtherapien und bietet für die Patientinnen weniger Behandlungsmöglichkeiten.

\section{Stress beschleunigt Metastasenbildung}

Um die Heterogenität zwischen Tumoren und Metastasen zu untersuchen, haben die Forschenden die Aktivität von Genen in einem Maus-Krebsmodell gemessen. Sie stellten fest, dass Metastasen eine erhöhte Aktivität bei den Glukokortikoid-Rezeptoren (GR) verzeichnen, die Stresshormone wie etwa Cortisol binden.
Mäuse mit Metastasen hatten höhere Konzentrationen von Stresshormonen als Mäuse ohne Metastasen. Die Studie zeigt, dass erhöhte Stresshormonlevel die GR aktivieren, was zu einer verstärkten Kolonisierung und Heterogenität der Krebszellen und letztlich zu einer verkürzten Lebensdauer führt.

\section{Reduzierte Wirkung der Chemotherapie}

Die GR binden auch synthetische Derivate von Cortisol wie etwa Dexamethason, das oft eingesetzt wird, um die Nebenwirkungen der Chemotherapie zu lindern. Die Forschungsgruppe zeigte, dass Dexamethason die Wirksamkeit des Medikaments Paclitaxel beeinträchtigen kann, welches vielfach als Chemotherapeutikum angewendet wird.

\section{FAZIT}

Diese Ergebnisse weisen darauf hin, dass bei der Verschreibung von Glukokortikoid-Hormonen an Patientinnen mit Brustkrebs Vorsicht geboten ist. Die Studie macht auch deutlich, dass die Blockierung der GR von Vorteil sein kann und zur Entwicklung neuer Therapien für die Bekämpfung der Metastasierung von Brustkrebs führen könnte.

Nach einer Mitteilung der Universität Base

Ernährung

\section{Krebspatienten profitieren von früher supplementie- render parenteraler Ernährung}

\footnotetext{
Caccialanza R et al. Early 7-day supplemental parenteral nutrition improves body composition and muscle strength in hypophagic cancer patients at nutritional risk. Supportive Care in Cancer 2019; 27: 2497-2506. doi:10.1007| s00520-018-4527-0
}

Viele Krebspatienten sind von Mangelernährung betroffen, die sich negativ auf das Überleben, den funktionellen Status sowie die Lebensqualität auswirkt. Bei solchen Patienten ist unter Umständen eine proaktive und intensive Nährstoffsupplementierung angezeigt. Riccardo Caccialanza et al. haben nun vor diesem Hintergrund die Effekte einer frühen 7-tägigen supplementierenden parenteralen Ernährung (SPE) untersucht.

Eingeschlossen in die Studie aus Italien waren 131 stationäre Patienten mit unterschiedlichen Krebsarten, Hypophagie und einem erhöhten Risiko für Unterernährung. Eine enterale Ernährung war bei dieser Patientengruppe nicht möglich. Die Studienteilnehmer erhielten ab einem frühen Zeitpunkt über 7 Tage hinweg eine SPE per Infusion ( $\bullet \mathbf{A b b}$. 1). Diese beinhaltete unter anderem Glukose, Aminosäuren, ggf. Lipide, Elektrolyte sowie einen Multivitaminund Multimineralmix. Parallel wurden die Patienten dazu angehalten, weiterhin oral Nahrung aufzunehmen. Es wurden demografische, klinische sowie anthropometrische Daten erhoben und der Nährstoffbedarf bestimmt. Bluttests umfassten unter anderem die Bestimmung des Blutzuckers sowie von $\mathrm{Na}, \mathrm{K}, \mathrm{Cl}, \mathrm{Ca}, \mathrm{Mg}$, P, Triglyzeriden, Cholesterin, ALT und CRP. Mittels der Bioimpedanz-Vektoranalyse (BIVA) wurde jeweils die Körperzusammensetzung bestimmt. Die Prüfärzte quantifizierten zudem die Greifkraft der Hand sowie das Präalbumin im Serum.

\section{Ergebnisse}

Für die finale Effektivitätsanalyse standen Daten von 118 Patienten zur Verfügung. Bei 102 Studienteilnehmern $(86,4 \%)$ lag eine Erkrankung im fortgeschrittenen Stadium vor. 101 Patienten $(85,6 \%)$ unterzogen sich einer Chemotherapie und 8 (6,8\%) einer Radiotherapie, 9 (7,6\%) erhielten ausschließlich eine unterstützende Betreuung. Bei 10 Patienten (8,5\%) wurde die SPE nach Tag 3 modifiziert. Im Fall von 33 Individuen (27,9\%) gelang es nicht, die Aufnahme von Kalorien und/oder Aminosäuren zu erhöhen. Nach Ablauf der 7-tägigen Intervention erreichten 90 der verbliebenen Patienten $(76,3 \%)$ den geschätzten Energie- und 


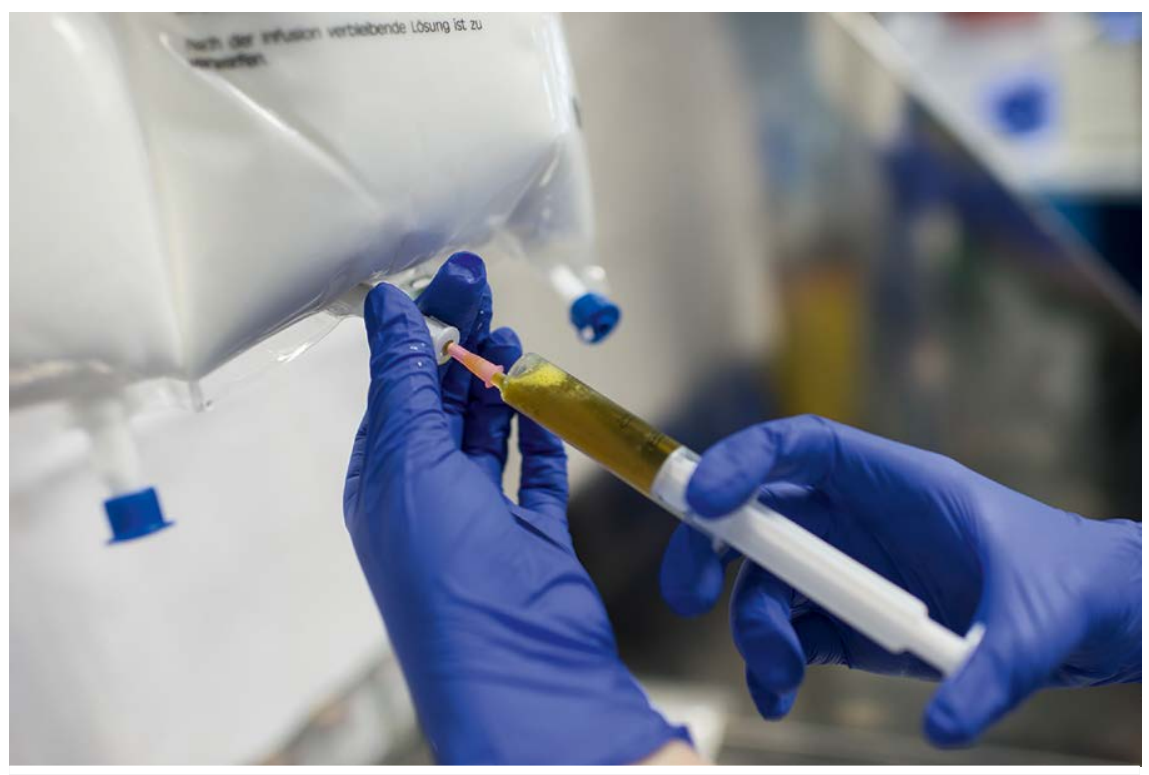

- Abb. 1 Bei vielen Krebspatienten tritt eine Mangelernährung auf, die sich dann negativ auf den Therapieverlauf auswirken kann. Eine frühzeitige supplementierende parenterale Ernährung kann dem entgegenwirken (Symbolbild). Quelle: Studio Blofield/Thieme Verlagsgruppe.

Proteinbedarf. Die Autoren identifizierten einen Zusammenhang zwischen der SPE und einer signifikanten Verbesserung der BIVA-Parameter (Phasenwinkel + 0,25; $p=0,001$, standardisierter Phasenwinkel $+0,33 ; p=0,002)$, der Greifkraft der Hand ( + 2,1 kg; $p<0,001$ ) sowie des Präalbumins im Serum (+3,8 mg/dl; $p<0,001)$.

Eine multivariable Analyse hatte folgendes Ergebnis: Patienten, bei denen der geschätzte Protein- und Kalorienbedarf gedeckt werden konnte, zeigten ausgeprägtere Effekte auf die BIVA-Parameter ( $\mathrm{n}=90 ; 76,3 \%$ ) (adjustierte Differenz: Phasenwinkel, +0,39; $\mathrm{p}=0,030$; standardisierter Phasenwinkel, +0,62; $p=0,009)$. Flüssigkeitsansammlungen waren am Ende der Studienperiode bei 11 Patienten (9,3\%) feststellbar, 4 (3,1\%) wiesen eine Hyperglykämie auf, 8 (6,1\%) eine Hypertriglyzeridämie und $1(0,8 \%)$ eine Hypokaliämie. Komplikationen aufgrund von Infektionen waren nicht zu verzeichnen.
Pankreas

\section{Kein Zusammenhang zwischen regelmäßiger Aspirin-Einnahme und Bauchspeicheldrüsenkrebs}

\author{
Khalaf $\mathrm{N}$ et al. Regular use of Aspirin or \\ non-Aspirin nonsteroidal anti-inflammatory \\ drugs is not associated with risk of incident \\ pancreatic cancer in two large cohorts. \\ Gastroenterology 2018; 154: 1380-1390. doi: \\ 10.1053/j.gastro.2017.12.001
}

Aspirin wird hauptsächlich zur Vorbeugung und Behandlung kardiovaskulärer Erkrankungen eingesetzt. Aktuell geht man davon aus, dass die Langzeiteinnahme auch das Risiko für Krebserkrankungen reduzieren könnte; dasselbe gilt für weitere nichtsteroidale Entzündungshemmer (NSAR). Ob die regelmäßige Einnahme von Aspirin und anderen NSAR auch das Risiko für Pankreaskrebs reduzieren kann, untersuchten amerikanische Ärzte nun in 2 großen Kohortenstudien.

Eine frühe SPE verbessert bei Krebspatienten die Körperzusammensetzung, die Greifkraft der Hand sowie die Serumspiegel von Präalbumin, so das Ergebnis der Studie. Als zentral für eine Verbesserung des Ernährungsstatus erwies sich eine vollständige Deckung des Kalorien- und Proteinbedarfs der Patienten. Nach Meinung der Autoren sollten weitere Studien die Effektivität dieser Intervention über mittlere bzw. lange Zeiträume - jeweils differenziert nach spezifischen Krebsarten - evaluieren.

Dr. Frank Lichert, Weilburg
Natalia Khalaf vom Brigham and Women's Hospital in Boston und Kollegen untersuchten prospektiv den Zusammenhang zwischen der Einnahme von Aspirin und NichtAspirin-NSAR und dem Auftreten eines Pankreaskarzinoms an 2 großen Kohorten über einen Zeitraum von 25 Jahren. Zusätzlich untersuchten sie den Zusammenhang zwischen der Plasmakonzentration von Salicylsäure, dem Hauptmetaboliten von Aspirin, vor Diagnose und dem künftigen Risiko für Bauchspeicheldrüsenkrebs.

Für die prospektive Studie rekrutierten die Ärzte Teilnehmer aus 2 US-amerikanischen Kohorten: Die Health-Professionals FollowUp-Study (HPFS) umfasst 51529 Männer im Alter zwischen 40 und 75 Jahren, die Nurse's-Health-Study (NHS) 121700 weibliche Pflegekräfte im Alter zwischen 30 und 55 Jahren. Mittels Fragebögen zu Studienbeginn und danach alle 2 Jahre wurde die Einnahme von Aspirin und Nicht-AspirinNSAR abgefragt (Dosierung, Häufigkeit). Als regelmäßig galt eine Einnahme von mindestens 2-mal pro Woche. Zusätzlich wurden Ernährungsgewohnheiten abgefragt und mittels des EDIP-Index ( $>$ Info) 
das entzündungsfördernde Potenzial der Ernährung ermittelt, um auch den Einfluss systemischer Entzündungen zu berücksichtigen. Um verschiedenen Zeitverzögerungen zwischen Einnahme und Krebsentwicklung Rechnung zu tragen, erfolgte eine Unterteilung in 4 Intervalle: 1. aktuell (Daten des zuletzt eingereichten Fragebogens), 2. Latenzzeit (6-8 Jahre Latenz zwischen Einnahme und Krebsdiagnose), 3. Ausgangswert (Daten des Ausgangs-Fragebogens), 4. kumulativ (Auswertung aller Fragebögen).

\section{EDIP}

EDIP steht für „empirical dietary inflammatory pattern” und ist ein neu entwickelter Maßstab für das entzündungsfördernde oder -hemmende Potenzial einer beliebigen Ernährungsweise. Entwickelt wurde er von den bekannten Bostoner Ernährungswissenschaftlern $\mathrm{Hu}$, Willett und Giovannucci mithilfe der Daten aus der Nurses Health Study. Er berücksichtigt die Verzehrsmenge von 18 Nahrungsmitteln (9 antiinflammatorische, 9 proinflammatorische) sowie die Konzentrationen von CRP, TNFaR2, IL-6 im Blut (J Nutr 2016; 146: 1560-1570).

\section{Keine signifikanten}

\section{Unterschiede}

Die Einschlusskriterien erfüllten 141940 Teilnehmer, von denen 32\% regelmäßig Aspirin einnahmen. Insgesamt entwickelten 1122 Teilnehmer ein Pankreaskarzinom über 4,2 Millionen Personenjahre. Weder die regelmäBige Einnahme von Aspirin noch von anderen NSAR erhöhte das Risiko, an Bauchspeicheldrüsenkrebs zu erkranken. Auch unter Berücksichtigung verschiedener Einnahmeintervalle zeigte sich kein Unterschied zwischen regelmäßiger und unregelmäßiger Einnahme von Aspirin oder anderen NSAR (Beispiel Aspirin, Anzahl neu aufgetretener Pankreaskarzinome: Einnahme „aktuell“, 529 vs. 593 Fälle; Einnahme mit Latenzzeit, 453 vs. 520 Fälle; „Ausgangswerte“, 353 vs. 524 Fälle). Auch bei Unterteilung in nichtreguläre Einnahme (Aspirin oder andere NSAR) und reguläre Einnahme von Aspirin alleine, Nicht-As-
pirin-NSAR alleine oder Kombination aus Aspirin und anderen NSAR ergaben sich keinerlei signifikante Unterschiede.

\section{Nur Diabetiker scheinen zu profitieren}

Lediglich in einer Subgruppen-Analyse zeigten sich eindeutige Unterschiede: Zum Zeitpunkt der Pankreaskarzinom-Diagnose litten 19\% (212 von 1122 Teilnehmern) an Diabetes. Diabetes-Patienten, die regelmäßig Aspirin nahmen, hatten ein beinahe $30 \% \mathrm{ge}$ ringeres Krebsrisiko als Patienten, die Aspirin nur unregelmäßig einnahmen. Ein derartiger Zusammenhang zeigte sich nicht bei regelmäßiger Einnahme von Nicht-AspirinNSAR. Interessanterweise hatten DiabetesPatienten mit einem sehr hohen EDIP-Index, also mit einer proinflammatorischen Ernährungsweise, ein niedrigeres Risiko, an Bauchspeicheldrüsenkrebs zu erkranken, wenn sie regelmäßig Aspirin einnahmen.

In der eingebetteten Fall-Kontroll-Studie untersuchten die Ärzte den Plasmagehalt von Salicylsäure vor einer Krebsdiagnose in 3 großen Kohorten. Durchschnittlich betrug die Zeit zwischen Blutabnahme und Krebsdiagnose 8 Jahre. 2 passende Kontrollteilnehmer $(n=784)$ wurden jedem Fall von Pankreaskarzinom ( $n=396$ ) zugeordnet. Die Ärzte fanden keine Assoziation zwischen dem Salicylsäure-Gehalt vor Diagnose und dem Risiko für Bauchspeicheldrüsenkrebs.

\section{FAZIT}

Die regelmäßige Einnahme von Aspirin oder anderen NSAR war nicht assoziiert mit einem erhöhten Risiko, an Bauchspeicheldrüsenkrebs zu erkranken. Die Studie beinhaltet mehrere analytische Ansätze, in denen die Autoren unterschiedlichste Zeitintervalle zwischen Aspirin-Einnahme und Krebsdiagnose berücksichtigen; in allen Ansätzen blieb das Fehlen einer Assoziation bestehen. Lediglich Patienten mit Diabetes scheinen unter Aspirin-Einnahme ein geringeres Pankreaskrebsrisiko zu haben.

Dr. Michaela Bitzer, Tübingen
Gesund leben

\section{Wie Schlaf das Immun- system stärkt}

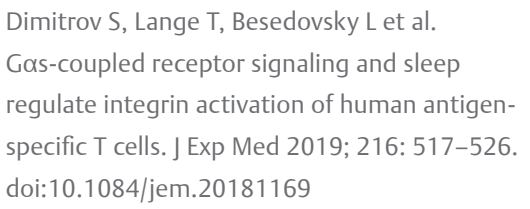

Wissenschaftler der Universitäten Tübingen und Lübeck haben in einer Studie einen Mechanismus nachgewiesen, über den Schlaf das Immunsystem fördert. Das Team konnte zeigen, dass bereits nach 3 Stunden ohne Schlaf die Funktion der T-Zellen beeinträchtigt war, die für die Bekämpfung von Erregern zuständig sind.

Die Wissenschaftler führten mit Probanden ein 24-stündiges Experiment durch: Eine Gruppe konnte nachts für 8 Stunden schlafen, eine zweite Gruppe blieb über den gesamten Zeitraum wach. Während des Experiments wurde den Teilnehmern regelmäßig Blut abgenommen. Dabei überprüfte das Forschungsteam die Bindungsstärke der T-Zellen an ein Molekül namens ICAM1 (intercellular adhesion molecule-1), das es ihnen ermöglicht, sich an andere infizierte Zellen anzuheften (Adhäsion), um sie anschließend zu beseitigen. Wie die Studie zeigt, war die Adhäsionsfähigkeit der T-Zellen bei den Probanden ohne Schlaf sichtlich reduziert. Um weiter zu untersuchen, wie Schlaf die T-Zellfunktion beeinflusst, wurde zudem Plasma von schlafenden sowie von wach gebliebenen Studienteilnehmern entnommen.

Dieses Plasma wurde für wenige Minuten auf isolierte T-Zellen gegeben. Stammte es von den Probanden ohne Schlaf, senkte es signifikant die Adhäsionsfähigkeit, verglichen mit dem Plasma der Probanden, die geschlafen hatten ( $>$ Abb. 2). Ein weiteres Experiment konnte zeigen, dass bereits bei kurzem Schlafentzug lösliche Stoffe die $\mathrm{G} \alpha_{\mathrm{s}}$-gekoppelten Rezeptoren aktivieren und die Adhäsion der T-Zellen beeinträchtigen.

Nach einer Mitteilung der Universität Lübeck, 12.2.2019 


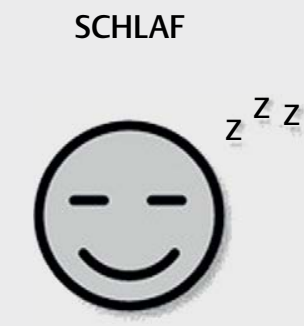

Plasmamediatoren (z.B. Adrenalin)

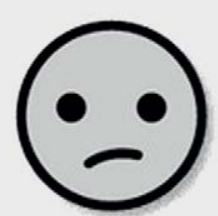

SCHLAFENTZUG
$\mathrm{G} \alpha_{\mathrm{S}}$-gekoppelter Rezeptor (z.B. für Adrenalin)

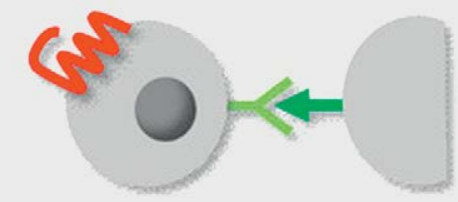
T-Zelle
ICAM-1

ADHÄSION $\uparrow$

Bindung
- Abb. 2 Schon drei Stunden Schlafentzug beeinträchtigt die Adhäsion der T-Zellen, also ihre Fähigkeit, sich an infizierte Zellen anzuheften und diese zu beseitigen. Grafik: Co-Autorin Priv. Doz. Dr. Tanja Lange mit freundlicher Genehmigung.
Pankreaskarzinom

\section{MRT zur Detektion von} Lebermetastasen bei potenziell resektablem duktalem Pankreaskarzinom

Marion-Audibert AM et al. Routine MRI with DWI sequences to detect liver metastases in patients with potentially resectable pancreatic ductal carcinoma and normal liver CT: A prospective multicenter study. Am J Roentgenol 2018; 211: W217-W225

Die Detektion kleiner Lebermetastasen stellt eine große Herausforderung beim Staging des duktalen Pankreaskarzinoms dar. Die thorako-abdomino-pelvine CT dient derzeit routinemäßig für das Staging; eine große Meta-Analyse zeigte aber, dass hier der Prädiktionswert zur Vorhersage der Resektabilität nur $81 \%$ beträgt; $19 \%$ der Patienten unterzogen sich also einer unnötigen Operation, hauptsächlich aufgrund von nicht identifizierten Lebermetastasen.
Die Kontrastauflösung im MRT und die Detektionsrate von Malignomen und fokalen Läsionen in der Leber sind besser als im CT. Dies gilt besonders für die Suche nach Läsionen mit einem Durchmesser von weniger als 1 Zentimeter bei Patienten mit duktalem Pankreaskarzinom. Von allen zur Verfügung stehenden MRT-Sequenzen scheint die diffusionsgewichtete Sequenz (DWI) hierbei die höchste Sensitivität zu besitzen. In einer prospektiven, multizentrischen Studie untersuchten Anne-Marie MarionAudibert vom Croix-Rousse-UniversityHospital in Lyon und Kollegen nun den Mehrwert einer systemischen, präoperativen MRT-Bildgebung zur Detektion von Lebermetastasen bei Patienten mit potenziell resektablem duktalem Pankreaskarzinom und unauffälligen Leberbefunden in $\operatorname{der}$ CT.

Patienten ( $n=118)$ mit duktalem Pankreaskarzinom, deren CT-Aufnahmen sowohl die Resektabilität des Karzinoms als auch eine unauffällige Leber bestätigten, konnten an der Studie teilnehmen. MRT-Aufnahmen ohne Kontrastmittel, DWI eingeschlossen, wurden $\leq 21$ Tage vor einer Operation gemacht. Die Auswertung von CT und MRT erfolgte unabhängig voneinander, mit einem Mindestabstand von 2 Wochen.
Mittels MRT fanden die Ärzte bei 16 Patienten $(13,8 \%)$ typische $(n=11)$ oder unklare $(n=5)$ Leberläsionen, die in der CT nicht sichtbar gewesen waren. Alle bis auf 1 Lebermetastase waren kleiner als $10 \mathrm{~mm}$. Bei $7 \mathrm{der}$ 11 Patienten mit typischen Leberläsionen wurde eine Malignität mit anschließender Biopsie gesichert; diese Patienten wurden nicht operiert. Bei den weiteren 9 Patienten erfolgte keine Biopsie, da die Läsionen im CT nicht sichtbar waren. Bei ihnen wurde eine explorative Chirurgie durchgeführt, genauso wie bei den 102 Patienten ohne Läsionen im MRT. Bei allen 5 Patienten mit unklaren Leberläsionen im MRT waren während der Operation keinerlei Läsionen detektierbar, allerdings fand sich bei 1 Patienten 6 Monate postoperativ doch noch eine Leberläsion, bei den 4 anderen nicht; insgesamt gab es somit 4 falsch positive Fälle (3,4\%). Dagegen fanden die Ärzte bei allen 4 Patienten mit typischen Leberläsionen im MRT ohne präoperative Biopsie intraoperativ Lebermetastasen. Bei 3 von 102 Patienten (2,9\%) mit unauffälligem MRT fanden sich intraoperativ Lebermetastasen (falsch negativ).

Die Sensitivität der MRT zur Detektion von typischen malignen Leberläsionen betrug $73,3 \%$, die Spezifizität $100 \%$ und $80,0 \%$ bzw. 96,1\%, wenn man die unklaren Fälle noch hinzurechnete. Der negative prädiktive Wert betrug insgesamt $97,1 \%$, der positive prädiktive Wert 75,0\%.

\section{FAZIT}

Verglichen mit der CT verbessert sich unter Einsatz von MRT mit DWI die Detektion von Lebermetastasen bei Patienten mit potenziell resektablem duktalem Pankreaskarzinom: 17 Lebermetastasen wurden bei 12/118 Patienten nur mit MRT detektiert, mit einer Sensitivität von $80 \%$; alle Fälle konnten histologisch bestätigt werden. Auch nach retrospektiver Analyse aller Bildgebungsdaten waren diese Läsionen im CT nicht sichtbar. Eine Operation konnte somit bei 7 Patienten vermieden werden, eine unnötige Pankreasresektion bei 4 Patienten.

Dr. Michaela Bitzer, Tübingen 